\title{
Higher organism load associated with failure of azithromycin to treat rectal chlamydia
}

\author{
F. Y. S. KONG ${ }^{1 *}$, S. N. TABRIZI ${ }^{2,3}$, C. K. FAIRLEY ${ }^{4}$, S. PHILLIPS ${ }^{3}$, \\ G. FEHLER ${ }^{4}$, M. LAW ${ }^{5}$, L. A. VODSTRCIL ${ }^{1,4}$, M. CHEN ${ }^{1,4}$, C. S. BRADSHAW ${ }^{1,4}$ \\ AND J.S. HOCKING ${ }^{1}$ \\ ${ }^{1}$ Melbourne School of Population and Global Health, University of Melbourne, Victoria, Australia \\ ${ }^{2}$ Department of Obstetrics and Gynaecology, University of Melbourne, Victoria, Australia \\ ${ }^{3}$ Royal Women's Hospital and Murdoch Children's Research Institute \\ ${ }^{4}$ Melbourne Sexual Health Centre, Alfred Health and Central Clinical School, Monash University, Victoria, \\ Australia \\ ${ }^{5}$ Kirby Institute, University of New South Wales, Sydney, Australia
}

Received 29 November 2015; Final revision 1 April 2016; Accepted 25 April 2016;

first published online 16 May 2016

\section{SUMMARY}

Repeat rectal chlamydia infection is common in men who have sex with men (MSM) following treatment with $1 \mathrm{~g}$ azithromycin. This study describes the association between organism load and repeat rectal chlamydia infection, genovar distribution, and efficacy of azithromycin in asymptomatic MSM. Stored rectal chlamydia-positive samples from MSM were analysed for organism load and genotyped to assist differentiation between reinfection and treatment failure. Included men had follow-up tests within 100 days of index infection. Lymphogranuloma venereum and proctitis diagnosed symptomatically were excluded. Factors associated with repeat infection, treatment failure and reinfection were investigated. In total, $227 \mathrm{MSM}$ were included 64 with repeat infections [28.2\%, 95\% confidence interval (CI) 22.4-34.5]. Repeat positivity was associated with increased pre-treatment organism load [odds ratio (OR) 1.7, 95\% CI 1.4-2.2]. Of 64 repeat infections, $29(12 \cdot 8 \%, 95 \%$ CI $8 \cdot 7-17 \cdot 8)$ were treatment failures and $35(15 \cdot 4 \%, 95 \%$ CI $11 \cdot 0-20 \cdot 8)$ were reinfections, $11(17 \cdot 2 \%, 95 \%$ CI 8.9-28.7) of which were definite reinfections. Treatment failure and reinfection were both associated with increased load (OR 2.0, 95\% CI $1 \cdot 4-2 \cdot 7$ and $1 \cdot 6,95 \%$ CI $1 \cdot 2-2 \cdot 2$, respectively). The most prevalent genovars were G, D and J. Treatment efficacy for $1 \mathrm{~g}$ azithromycin was $83 \cdot 6 \%(95 \%$ CI $77 \cdot 2-88 \cdot 8)$. Repeat positivity was associated with high pre-treatment organism load. Randomized controlled trials are urgently needed to evaluate azithromycin's efficacy and whether extended doses can overcome rectal infections with high organism load.

Key words: Chlamydia trachomatis, genovar, organism load, rectal, repeat infection.

\section{INTRODUCTION}

Chlamydia trachomatis is the most common bacterial sexually transmitted infection (STI) worldwide [1]. In

* Author for correspondence: Mr F. Y. S. Kong, Melbourne School of Population and Global Health, University of Melbourne, Level 3, 207 Bouverie St, Melbourne, Australia 3004. (Email: kongf@unimelb.edu.au)
Australia, an estimated $40 \%$ of diagnoses are in men [2]; the prevalence of rectal chlamydia is $5 \cdot 6 \%$ in men who have sex with men (MSM) and infections are associated with an increased risk of HIV transmission [3].

Current guidelines for MSM from the Center for Disease Control and Prevention recommend that all positive uncomplicated chlamydia infections be 
treated with a single $1 \mathrm{~g}$ dose of azithromycin or 7 days (100 mg twice daily) of doxycycline [4]. However, there is increasing concern about rectal chlamydia treatment failure with about $22 \%$ of MSM presenting with repeat chlamydia infection following treatment with azithromycin [5]. In response to these concerns, European [6] and Australian [7] guidelines have been recently revised and now recommend rectal infections be treated with 7 days doxycycline as first-line treatment. It is important to note, however, that there are only observational studies and no randomized controlled trials (RCTs) comparing azithromycin with doxycycline for the treatment of rectal chlamydia, so the level of evidence supporting this recommendation is limited.

High organism load has been associated with the failure of azithromycin to treat genital chlamydia in studies of women [8] and trachoma [9]. However, there are few data available regarding the association between rectal chlamydia treatment failure and organism load. What is known is that higher organism loads are reported at the rectal site compared to other genital sites [10] and that chlamydia isolates demonstrating heterotypic resistance to macrolides in vitro have been reported [11] at high, but not low, levels of organism load [12].

This study aimed to estimate the risk of repeat rectal chlamydia infection in MSM and to investigate the association between organism load and repeat rectal infection following treatment. We also investigated rectal chlamydia genovar distribution in MSM and calculated the efficacy for those treated with $1 \mathrm{~g}$ azithromycin.

\section{METHODS}

\section{Study participants, inclusion and exclusion criteria, data collection}

This is a retrospective study of stored samples from asymptomatic MSM attending Melbourne Sexual Health Centre (MSHC, Australia) between July 2008 and October 2013 who had a follow-up test result within 100 days of an initial (index) rectal chlamydia infection. At MSHC, all rectal swab samples are clinician-collected without the aid of an anoscopy. All chlamydia-positive rectal swab samples were tested using the BD strand displacement amplification (SDA) test (BD ProbeTec; Becton, Dickinson and Company, USA) and were subsequently stored in BD transport medium at $-80^{\circ} \mathrm{C}$ for research purposes. For individuals who had multiple positive tests during the study period, only the most recent test/retest samples were included. Men diagnosed symptomatically for lymphogranuloma venereum (LGV) or proctitis was excluded as these patients would have been treated with extended treatment courses. At MSHC, LGV is treated with 3 weeks doxycycline (100 mg twice daily) and proctitis is treated with combination therapy using a single dose of $1 \mathrm{~g}$ azithromycin, 3 weeks doxycycline $(100 \mathrm{mg}$ twice daily), $500 \mathrm{mg}$ ceftriaxone and $500 \mathrm{mg}$ valaciclovir twice daily for 7-10 days. Any asymptomatic LGV cases detected as a result of this study were included in the analysis because these cases were originally managed as uncomplicated chlamydia infection as is clinical practice for any asymptomatic rectal chlamydia infection in the absence of genotyping. Electronic patient data were extracted for individuals. This included the patient's age, treatment received at the time of the initial diagnosis, date of drug prescription, co-infections with other STIs including HIV, past STIs, sexual risk behaviour and presence of any rectal symptoms. The time between treatment and repeat testing was estimated based on the date of the actual drug prescription.

\section{Testing, organism load, genovar and multilocus sequence typing (MLST) testing}

Stored chlamydia SDA-positive rectal samples were sent to the Department of Microbiology and Infectious Diseases, at the Royal Women's Hospital, Melbourne, Australia for chlamydial bacterial load, genovar and MLST testing.

\section{DNA extraction}

A $200 \mu 1$ aliquot was extracted by using the automated system, MagNA Pure 96 (Roche Applied Science, Germany) according to the manufacturer's instructions. The total nucleic acid was then eluted in a final volume of $100 \mu 1$ in MagNA Pure 96 elution buffer.

\section{Chlamydia genovar and bacterial load}

Aliquots of $5 \mu \mathrm{l}$ of the extracted nucleic acid was utilized in each qPCR assay for determination of bacterial load and chlamydial genovar as described previously [13]. The chlamydial load in each tested sample was quantified by comparing the crossing threshold of each sample to the crossing threshold of a standard curve constructed by amplifying different 
Table 1. Algorithm to differentiate between reinfection and treatment failure

\begin{tabular}{llll}
\hline \hline $\begin{array}{l}\text { Genovar: index } v s . \\
\text { follow-up result }\end{array}$ & $\begin{array}{l}\text { Had sex in past } \\
\text { months* }\end{array}$ & $\begin{array}{l}\text { Condomless sex in } \\
\text { past } 3 \text { months } \dagger\end{array}$ & Outcome \\
\hline Different & Not relevant & Not relevant & Re-infection \\
Same & Yes & Yes & Re-infection \\
Same & Yes & No data available & Re-infection \\
No data available & Yes & No data available & Re-infection \\
Same & No & No data available & Treatment failure \\
Same & Yes & No & Treatment failure \\
No data available & No & No data available & Treatment failure \\
\hline \hline
\end{tabular}

* Reported had male sex partners in the last 3 months.

$\uparrow$ Reported specifically had condomless sex as a receptive partner in the last 3 months.

known copy numbers of the ompl gene. In addition, an aliquot of DNA was amplified for $\beta$-globin gene as an internal control to assess sampling adequacy. Results were calculated in copies $/ \mathrm{ml}$ and were logtransformed for analysis.

\section{MLST analysis}

For individuals with two sequential positive samples with identical genovar, MLST was performed to help differentiate between treatment failure and reinfection by evaluating any sequence variation across five genes, i.e. CT144, CT058, CT172, $p b p B$ and $h c t B$ [14] and compared to an online MLST database (http://mlstdb.bmc.uu.se/).

\section{Outcome definition}

Our primary outcome was repeat rectal chlamydia infection diagnosed by SDA test within 100 days of diagnosis and treatment for an index rectal chlamydia infection. For the secondary outcome, we further classified a repeat rectal chlamydia infection as a treatment failure or reinfection using both the genovar and reported sexual behaviour (Table 1). Our classification of treatment failure is conservative; all repeat infections of the same genovar in individuals who report condomless sex, are classified as reinfection.

\section{Statistical methods}

Descriptive statistics were used to describe the characteristics of men participating. The proportion and $95 \%$ confidence intervals (CIs) of men who had a repeat positive diagnosis was calculated using exact binomial methods. Our primary outcome was analysed using univariate and multivariate logistic regression to investigate factors associated with repeat positivity including organism load, patient demographics, sexual risk behaviour, treatment received, and concurrent infections with other STIs. Variables included in our multivariate models were selected on the basis of clinical relevance and the likelihood ratio test. Our secondary outcome was analysed using univariate multinomial regression to investigate factors associated with treatment failure and reinfection. No multivariate analysis was undertaken due to the small number of treatment failures and reinfections. We also conducted a sensitivity analysis to exclude cases that were tested within 28 days of receiving treatment because of the possibility of false-positive results [4]. Box plots were generated to compare distributions in organism load between those who did or did not have a repeat infection and by their OMP classification - the difference compared using Wilcoxon ranksum tests. Azithromycin efficacy was calculated as the proportion of individuals testing SDA negative for rectal chlamydia when retested within 100 days after receiving treatment after excluding those classified as reinfections. Analysis was performed with Stata v. 13.0 (StataCorp., USA).

\section{Ethics statement}

Ethical approval for this study was granted by the Alfred Hospital Ethics Committee (373/13).

\section{RESULTS}

A total of 227 MSM were included in the study, of whom $64(28 \cdot 2 \%)$ presented with a repeat chlamydiapositive diagnosis within 100 days, giving a total of 291 chlamydia-positive samples available for further laboratory analysis. Of these 291 samples, genovar and organism load was able to be determined for $272(93 \cdot 4 \%)$. 
Table 2. Patient characteristics in index cases

\begin{tabular}{|c|c|}
\hline Characteristic & $n(\%)$ \\
\hline \multicolumn{2}{|l|}{ Age (years) } \\
\hline$\leqslant 29$ & $125(55 \cdot 1)$ \\
\hline $30-39$ & $59(26 \cdot 0)$ \\
\hline$\geqslant 40$ & $43(18 \cdot 9)$ \\
\hline Organism load $\left(\log _{10}\right.$ copies $\left./ \mathrm{ml}\right)$ & Median $3 \cdot 8$ \\
\hline $\begin{array}{l}\text { Time between treatment and diagnosis } \\
\text { (days) }\end{array}$ & Median 55 \\
\hline \multicolumn{2}{|l|}{$\begin{array}{l}\text { Concurrent infections with other STI } \\
\text { (excluding HIV) }\end{array}$} \\
\hline Chlamydia, urine & $36(15 \cdot 9)$ \\
\hline Gonorrhoea, rectal & $27(11 \cdot 9)$ \\
\hline Syphilis & $6(2 \cdot 6)$ \\
\hline \multicolumn{2}{|l|}{ HIV status } \\
\hline Positive & $45(19 \cdot 8)$ \\
\hline Negative & $182(80 \cdot 2)$ \\
\hline \multicolumn{2}{|l|}{ Ever had chlamydia in past } \\
\hline Yes & $29(12 \cdot 8)$ \\
\hline No & $198(87 \cdot 2)$ \\
\hline \multicolumn{2}{|l|}{ No. of male partners last 3 months } \\
\hline $0-1$ & $79(34 \cdot 8)$ \\
\hline$\geqslant 2$ & $148(65 \cdot 2)$ \\
\hline \multicolumn{2}{|l|}{ No. of male partners last 12 months } \\
\hline $0-1$ & $60(26 \cdot 4)$ \\
\hline $2-5$ & $56(24 \cdot 7)$ \\
\hline$\geqslant 6$ & $111(48 \cdot 9)$ \\
\hline \multicolumn{2}{|l|}{$\begin{array}{l}\text { Condom use with male partner, receptive } \\
\text { anal intercourse, last } 3 \text { months }\end{array}$} \\
\hline Always & $48(24 \cdot 9)$ \\
\hline Never/sometimes & $49(25 \cdot 4)$ \\
\hline No sex last 3 months & $96(49 \cdot 7)$ \\
\hline \multicolumn{2}{|l|}{ Serovar } \\
\hline G & $76(34 \cdot 9)$ \\
\hline $\mathrm{D}$ & $64(29 \cdot 4)$ \\
\hline $\mathrm{J}$ & $35(16 \cdot 1)$ \\
\hline $\mathrm{E}$ & $23(10 \cdot 6)$ \\
\hline $\mathrm{B}$ & $8(3 \cdot 7)$ \\
\hline L2 (LGV) & $7(3 \cdot 2)$ \\
\hline $\mathrm{F}$ & $5(2 \cdot 3)$ \\
\hline \multicolumn{2}{|l|}{ Genovar by omp classification } \\
\hline B complex (B, D, E, L2) & $102(44 \cdot 9)$ \\
\hline Intermediate group $(F, G)$ & $81(35 \cdot 7)$ \\
\hline $\mathrm{C}$ complex $(\mathrm{H}, \mathrm{I}, \mathrm{J} \text { or } \mathrm{K})^{*}$ & $35(15 \cdot 4)$ \\
\hline \multicolumn{2}{|l|}{ Treatment outcome } \\
\hline Treatment success & $163(71 \cdot 8)$ \\
\hline Repeat infection & $64(28 \cdot 2)$ \\
\hline Treatment failure $\dagger$ & $29(12 \cdot 8)$ \\
\hline Reinfection $\dagger$ & $35(15 \cdot 4)$ \\
\hline
\end{tabular}

* Only $\mathrm{J}$ found in this study in $\mathrm{C}$ complex.

$\dagger$ See Table 1 for classification algorithm.

\section{Characteristics of men}

The median age of participants at first diagnosis was 29 (range 18-78) years (Table 2). Concurrent infections with urethral chlamydia, rectal gonorrhoea and syphilis infection were reported in about $16 \%, 12 \%$ and $3 \%$, respectively; $20 \%$ were HIV positive. Overall about $65 \%$ reported $\geqslant 2$ partners in the last 3 months and $25 \%$ reported always using a condom during the last 3 months. The median time between receiving treatment and the follow-up test was 55 days, and $50 \%$ of men were retested 36-71 days after receiving treatment.

\section{Genovar and genotyping (MLST)}

For index cases, genovar was determined for $218(96 \cdot 0 \%)$ men; the most commonly detected genovar was $\mathrm{G}$ $(34 \cdot 9 \%)$, followed by D (29.4\%), J (16.1\%), E (10.6\%), B $(3 \cdot 7 \%)$ and F $(2 \cdot 3 \%)$. A total of seven $(3 \cdot 2 \%)$ men were found to have asymptomatic LGV-associated genotypes on their index swab that were missed during their initial diagnosis. Of men presenting with a repeat infection, $46(71.8 \%)$ presented with an identical genovar, two $(3 \cdot 2 \%)$ with a different genovar and $16(25 \cdot 0 \%)$ were not assessable. Different sequence types (using MLST) were seen in 9/46 (19.6\%) patients, suggesting new infections. These results show that overall 11 cases $(17 \cdot 2 \%, 95 \%$ CI $8 \cdot 9-28 \cdot 7)$ were definite reinfections on the basis of genovar analysis.

\section{Organism load}

Median organism load was higher for repeat infection cases than for index cases $\left(4 \cdot 4 \log _{10}\right.$ copies $/ \mathrm{ml}$ vs. $3 \cdot 8$ $\log _{10}$ copies/ml, $P=0 \cdot 03$ ) (Fig. 1).

Median organism load in the index infection was significantly higher in men who had a subsequent treatment failure $\left(n=29,5 \cdot 2 \log _{10}\right.$ copies $/ \mathrm{ml}, P<$ $0 \cdot 01)$ or reinfection $\left(n=35,4 \cdot 7 \log _{10}\right.$ copies $/ \mathrm{ml}, P<$ $0 \cdot 01)$ compared to men whose infection was successfully treated ( $n=163,3.5 \log _{10}$ copies $\left./ \mathrm{ml}\right)$ (Fig. 2). There was no difference in organism load for the index case between men who had treatment failure or reinfection on retest $(P=0 \cdot 35)$.

Median organism load was significantly higher for $\mathrm{B}$ complex than C complex genovars $\left(4 \cdot 1\right.$ vs. $3 \cdot 4 \log _{10}$ copies $/ \mathrm{ml}, P<0.01)$ but not between Intermediate and $\mathrm{C}$ complex genovars $\left(3.9\right.$ vs. $3.4 \log _{10}$ copies $/ \mathrm{ml}$, $P=0 \cdot 10)$ or between Intermediate and B complex genovars (3.9 vs. $4.1 \log _{10}$ copies $/ \mathrm{ml}, P=0 \cdot 11$ ) (Fig. 3).

\section{Repeat positive diagnosis}

A total of $64(28 \cdot 2 \%, 95 \%$ CI $22 \cdot 4-34 \cdot 5)$ men had a rectal chlamydia diagnosis with a repeat positive result. Univariate analysis found only increased organism 


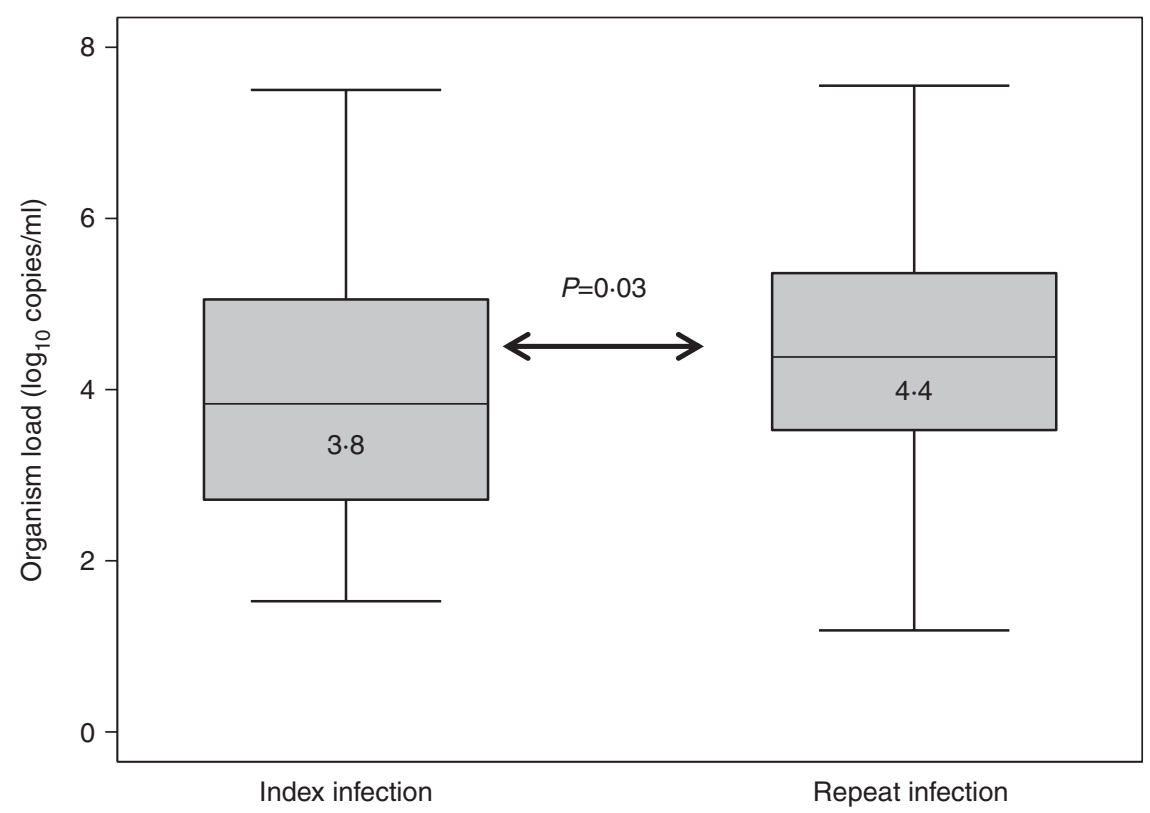

Fig. 1. Organism load $/ \mathrm{ml}$ between index and repeat infections.

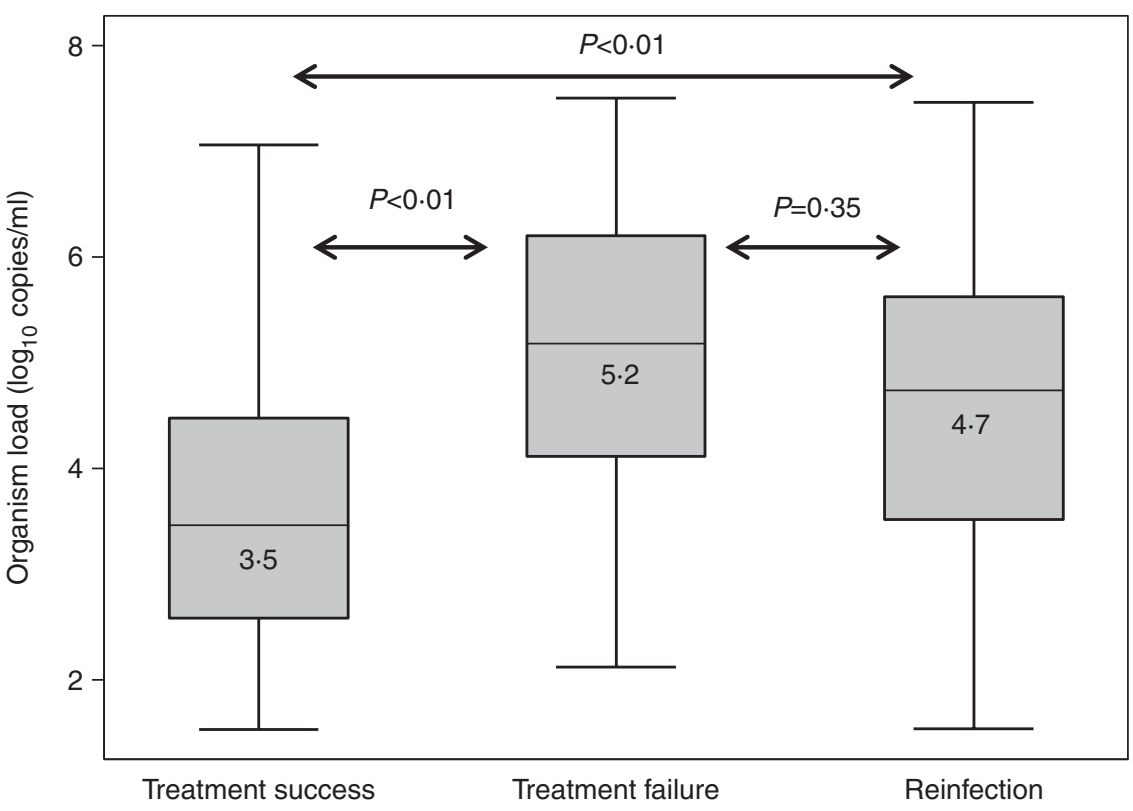

Fig. 2. Organism load/ml (in index cases) by outcome.

load [odds ratio (OR) $1 \cdot 8,95 \%$ CI 1·4-2·3] was significantly associated with a repeat positive result (Table 3 ).

Multivariate analysis (including age, organism load, time to next test, HIV status and number of recent partners) found repeat positivity was associated with increased organism load (OR 1·7, 95\% CI $1 \cdot 4$ $2 \cdot 2)$ and $\geqslant 2$ sexual partners in the last 3 months (OR 2.5, 95\% CI 1·1-5.8). Of the seven men found to have LGV, one (14\%) was diagnosed with a repeat rectal chlamydia infection. There was no difference in the proportion with repeat positive chlamydia by index serovar $(P=0 \cdot 14)$ (Table 3$)$.

Of the 64 men with repeat infection, an estimated $29(12 \cdot 8 \%, 95 \%$ CI $8 \cdot 7-17 \cdot 8)$ were classified as treatment failure and $35(15 \cdot 4 \%, 95 \%$ CI $11 \cdot 0-20 \cdot 8)$ as reinfection. Univariate analysis found that increased organism load was associated with treatment failure (OR 2.0, 95\% CI 1.4-2.7) or reinfection (OR 1.6, 


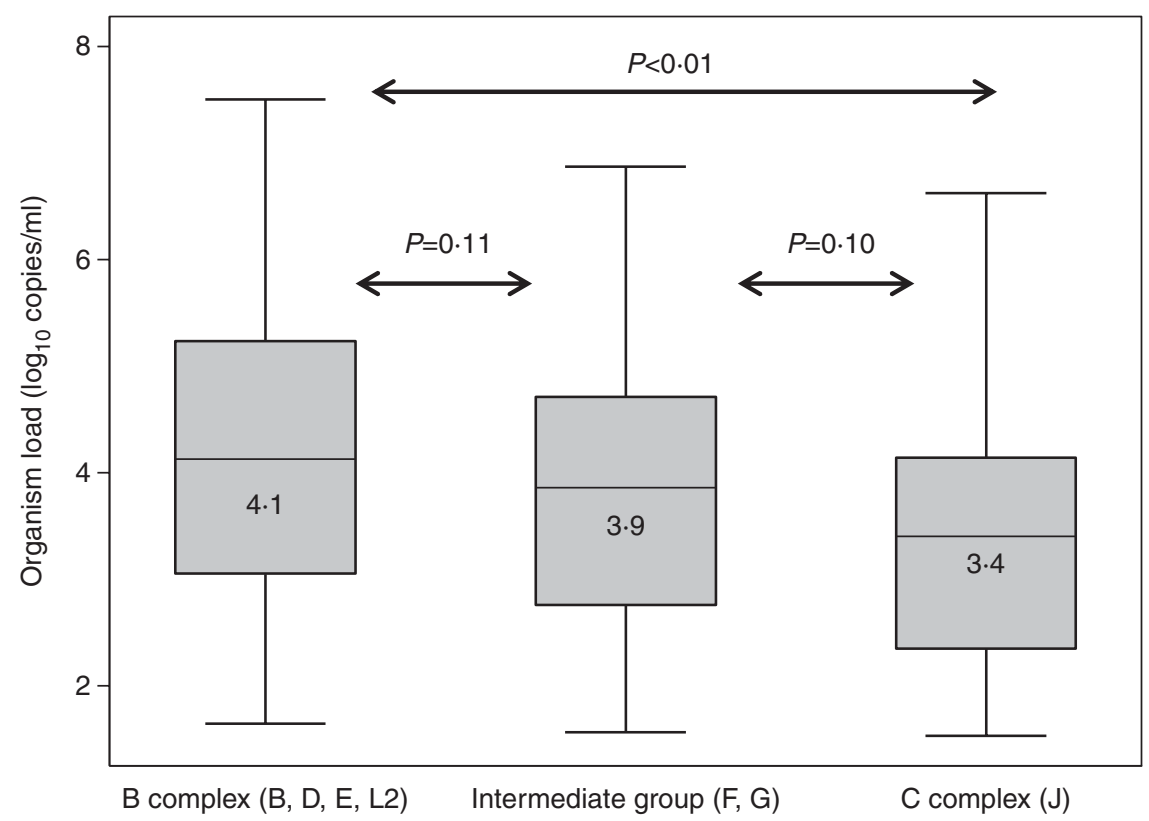

Fig. 3. Organism load/ml (in index cases) by OMP classification.

$95 \%$ CI $1 \cdot 2-2 \cdot 2)$. No other variables were associated with either outcome.

\section{Sensitivity analysis}

A total of 30 men were retested within 28 days of receiving treatment of whom seven $(23.3 \%)$ were repeat positives: four $(13 \cdot 3 \%)$ and three $(10 \cdot 0 \%)$ being treatment failures or reinfection. Excluding those who were tested within 28 days of receiving treatment we found that $(a)$ the number of partners in the past 3 months was no longer associated with repeat positive infections in multivariate analysis, and $(b)$ there was no effect on the association of organism load on treatment failure or reinfection in univariate analysis.

\section{Treatment efficacy}

Treatment records were available for $220(96 \cdot 9 \%)$ index cases with $203(92.3 \%)$ being prescribed a single $1 \mathrm{~g}$ dose of azithromycin; other treatments were prescribed for the other 17 cases. Of those treated with $1 \mathrm{~g}$ azithromycin and excluding those classified as reinfection $(n=32), 143$ were effectively cured at follow-up corresponding to a treatment efficacy for $1 \mathrm{~g}$ azithromycin of $83 \cdot 6 \%$ (143/ $171,95 \%$ CI $77 \cdot 2-88 \cdot 8)$. Of the seven missed cases of LGV, four were successfully treated with $1 \mathrm{~g}$ azithromycin, two successfully treated with combination of doxycycline and azithromycin, and only one case reported as a treatment failure with $1 \mathrm{~g}$ azithromycin.

\section{DISCUSSION}

We found that repeat rectal chlamydia was common in MSM with $>28 \%$ being diagnosed with a repeat rectal chlamydia infection within 100 days of treatment. While new infections or reinfection accounted for a significant proportion of the repeat infections, we found that $12 \cdot 8 \%$ were likely to represent probable treatment failure on the basis that they were the same genovar and had either not had sex or had only protected anal sex. We also found that high rectal chlamydia organism load is associated with repeat infection with a twofold increase in the odds of treatment failure.

We found an estimated treatment efficacy of $83.6 \%$ following treatment with a single $1 \mathrm{~g}$ dose of azithromycin which is similar to the $85 \%$ treatment efficacy estimate from a recent meta-analysis of rectal chlamydia treatments [5]. An efficacy of $83.6 \%$ is less than the $95 \%$ efficacy recommended by the World Health Organization [15] and raises the question of whether $1 \mathrm{~g}$ azithromycin is the optimal treatment for rectal chlamydia. Guidelines for the treatment of rectal chlamydia do vary with doxycycline being the recommended first-line treatment in the EU [6] and Australia [7], but azithromycin is the first-line treatment in the USA [4]. However, there are no RCTs comparing 7-day doxycycline and $1 \mathrm{~g}$ azithromycin treatments for rectal chlamydia and this higher level evidence is urgently needed given the difficultly of interpreting observational studies. Additionally, no 
Table 3. Factors associated with a repeat positive rectal chlamydia diagnosis, treatment failure and reinfection*

\begin{tabular}{|c|c|c|c|c|c|c|c|}
\hline \multirow[b]{2}{*}{ Characteristic } & \multicolumn{3}{|c|}{ Repeat positive $(n=64)$} & \multicolumn{2}{|c|}{ Treatment failure $(n=29)$} & \multicolumn{2}{|c|}{ Reinfection $(n=35)$} \\
\hline & $n(\%)$ & $\begin{array}{l}\text { Unadjusted OR } \\
(95 \% \mathrm{CI})\end{array}$ & $\begin{array}{l}\text { Adjusted OR } \\
(95 \% \mathrm{CI})\end{array}$ & $n(\%)$ & $\begin{array}{l}\text { Unadjusted OR } \\
(95 \% \mathrm{CI})\end{array}$ & $n(\%)$ & $\begin{array}{l}\text { Unadjusted OR } \\
(95 \% \mathrm{CI})\end{array}$ \\
\hline \multicolumn{8}{|l|}{ Age (years) } \\
\hline$\leqslant 29$ & $38(59 \cdot 4)$ & Ref. & Ref. & $18(62 \cdot 1)$ & Ref. & $20(57 \cdot 1)$ & Ref. \\
\hline $30-39$ & $16(25 \cdot 0)$ & $0 \cdot 9(0 \cdot 4-1 \cdot 7)$ & $0 \cdot 9(0 \cdot 4-2 \cdot 1)$ & $8(27 \cdot 6)$ & $0 \cdot 9(0 \cdot 4-2 \cdot 2)$ & $8(22 \cdot 9)$ & $0 \cdot 8(0 \cdot 3-2 \cdot 0)$ \\
\hline$\geqslant 40$ & $10(15 \cdot 6)$ & $0 \cdot 7(0 \cdot 3-1 \cdot 8)$ & $0 \cdot 7(0 \cdot 3-1 \cdot 8)$ & $3(10 \cdot 3)$ & $0 \cdot 4(0 \cdot 1-1 \cdot 6)$ & $7(20 \cdot 0)$ & $0 \cdot 9(0 \cdot 4-2 \cdot 4)$ \\
\hline Organism load in index cases $\left(\log _{10}\right.$ copies $\left./ \mathrm{ml}\right)$ & Median 5.0 & $1 \cdot 8(1 \cdot 4-2 \cdot 3) \dagger$ & $1 \cdot 7(1 \cdot 4-2 \cdot 2) \dagger$ & Median $5 \cdot 2$ & $2 \cdot 0(1 \cdot 4-2 \cdot 7) \dagger$ & Median $4 \cdot 7$ & $1 \cdot 6(1 \cdot 2-2 \cdot 2) \dagger$ \\
\hline Time between treatment and diagnosis (days) & Median 54 & $1 \cdot 0(0 \cdot 9-1 \cdot 1)$ & $1 \cdot 0(0 \cdot 9-1 \cdot 1)$ & Median 55 & $1 \cdot 0(0 \cdot 9-1 \cdot 2)$ & Median 50 & $1 \cdot 1(0 \cdot 9-1 \cdot 2)$ \\
\hline \multicolumn{8}{|l|}{$\begin{array}{l}\text { Concurrent co-infections with other STI } \\
\text { (excluding HIV) }\end{array}$} \\
\hline Yes & $15(23 \cdot 4)$ & $0 \cdot 7(0 \cdot 3-1 \cdot 3)$ & Excluded & $6(20 \cdot 7)$ & $0 \cdot 6(0 \cdot 2-1 \cdot 5)$ & $9(25 \cdot 7)$ & $0 \cdot 7(0 \cdot 3-1 \cdot 7)$ \\
\hline No & $49(76 \cdot 6)$ & Ref. & & $23(79 \cdot 3)$ & Ref. & $26(74 \cdot 3)$ & Ref. \\
\hline \multicolumn{8}{|l|}{ Ever had chlamydia in past } \\
\hline Yes & $11(17 \cdot 2)$ & $1 \cdot 7(0 \cdot 7-3 \cdot 8)$ & Excluded & $4(13 \cdot 8)$ & $1 \cdot 3(0 \cdot 4-4 \cdot 1)$ & $7(20 \cdot 0)$ & $2 \cdot 0(0 \cdot 8-5 \cdot 3)$ \\
\hline No & $53(82 \cdot 8)$ & Ref. & & $25(86 \cdot 2)$ & Ref. & $28(80 \cdot 0)$ & Ref. \\
\hline \multicolumn{8}{|l|}{ HIV status } \\
\hline Positive & $13(20 \cdot 3)$ & $1 \cdot 0(0 \cdot 5-2 \cdot 2)$ & $2 \cdot 0(0 \cdot 7-5 \cdot 2)$ & $6(20 \cdot 7)$ & $1 \cdot 1(0 \cdot 4-2 \cdot 8)$ & $7(20 \cdot 0)$ & $1 \cdot 0(0 \cdot 4-2 \cdot 6)$ \\
\hline Negative & $51(79 \cdot 7)$ & Ref. & Ref. & $23(79 \cdot 3)$ & Ref. & $28(80 \cdot 0)$ & Ref. \\
\hline \multicolumn{8}{|l|}{ No. of male partners last 3 months } \\
\hline $0-1$ & $16(25 \cdot 0)$ & Ref. & Ref. & $8(27 \cdot 6)$ & Ref. & $8(22 \cdot 9)$ & Ref. \\
\hline$\geqslant 2$ & $48(75 \cdot 0)$ & $1 \cdot 9(1 \cdot 0-3 \cdot 6)$ & $2 \cdot 5(1 \cdot 1-5 \cdot 8) \dagger$ & $21(72 \cdot 4)$ & $1 \cdot 7(0 \cdot 7-4 \cdot 0)$ & $27(77 \cdot 1)$ & $2 \cdot 1(0 \cdot 9-5 \cdot 0)$ \\
\hline \multicolumn{8}{|l|}{ No. of male partners last 12 months } \\
\hline $0-1$ & $12(18 \cdot 8)$ & Ref. & Excluded & $6(20 \cdot 7)$ & Ref. & $6(17 \cdot 1)$ & Ref. \\
\hline $2-5$ & $17(26 \cdot 6)$ & $1 \cdot 7(0 \cdot 7-4 \cdot 1)$ & & $6(20 \cdot 7)$ & $1 \cdot 2(0 \cdot 4-4 \cdot 1)$ & $11(31 \cdot 4)$ & $2 \cdot 3(0 \cdot 8-6 \cdot 6)$ \\
\hline$\geqslant 6$ & $35(54 \cdot 7)$ & $1 \cdot 8(0 \cdot 9-3 \cdot 9)$ & & $17(58 \cdot 6)$ & $1 \cdot 8(0 \cdot 7-4 \cdot 9)$ & $18(51 \cdot 4)$ & $1 \cdot 9(0 \cdot 7-5 \cdot 1)$ \\
\hline \multicolumn{8}{|l|}{$\begin{array}{l}\text { Condom use with male partner, receptive anal } \\
\text { intercourse, last } 3 \text { months }\end{array}$} \\
\hline Always & $16(28 \cdot 1)$ & Ref. & Excluded & $6(21 \cdot 4)$ & Ref. & $10(34 \cdot 5)$ & Ref. \\
\hline Never/sometimes & $16(28 \cdot 1)$ & $1 \cdot 0(0 \cdot 4-2 \cdot 3)$ & & $8(28 \cdot 6)$ & $1 \cdot 3(0 \cdot 5-3 \cdot 1)$ & $8(27 \cdot 6)$ & $1 \cdot 2(0 \cdot 5-2 \cdot 9)$ \\
\hline Missing/not applicable & $25(43 \cdot 9)$ & $0 \cdot 7(0 \cdot 3-1 \cdot 5)$ & & $14(50 \cdot 0)$ & - & $11(37 \cdot 9)$ & - \\
\hline \multicolumn{8}{|l|}{ Genovar by OMP classification } \\
\hline B complex (B, D, E, L2) & $30(52 \cdot 6)$ & Ref. & Excluded & $13(50 \cdot 0)$ & Ref. & $17(57 \cdot 8)$ & Ref. \\
\hline Intermediate group $(\mathrm{F}, \mathrm{G})$ & $19(33 \cdot 3)$ & $0 \cdot 7(0 \cdot 4-1 \cdot 4)$ & & $12(46 \cdot 2)$ & $1 \cdot 1(0 \cdot 5-2 \cdot 5)$ & $7(22 \cdot 6)$ & $0 \cdot 5(0 \cdot 2-1 \cdot 2)$ \\
\hline C complex $(\mathrm{J})$ & $8(14 \cdot 0)$ & $0 \cdot 7(0 \cdot 3-1 \cdot 7)$ & & $1(3 \cdot 9)$ & $0 \cdot 2(0 \cdot 1-0 \cdot 3)$ & $7(22 \cdot 6)$ & $1 \cdot 1(0 \cdot 1-0 \cdot 4)$ \\
\hline
\end{tabular}

OR, Odds ratio; CI, confidence interval.

$*$ Treatment success is the reference group $(n=163)$; Excluded $=$ excluded from multivariate analysis.

$\dagger P<0.05$. 
pharmacokinetic data are available on azithromycin in rectal tissue so it remains uncertain if rectal tissue concentrations are sufficient to effectively treat chlamydia. However, available data on azithromycin concentrations in gastric tissue (a proxy for rectal tissue) report high concentrations in gastric tissue, juice and mucus $[16,17]$ raising the possibility that factors other than tissue concentrations may impact treatment efficacy [18].

To our knowledge, these are the first available data showing the association between higher organism load and repeat rectal chlamydia infections, but this has been observed in other infection sites including the eye [9], vagina [8] and throat [19]. One possible explanation for this finding is potential heterotypic resistance occurring in rectal infections whereby a chlamydia infection may comprise both treatmentsusceptible and less-susceptible organisms. If the organism load is sufficiently high, the less susceptible organisms can survive treatment thereby allowing the infection to persist once treatment is complete. Heterotypic resistance has been demonstrated in vitro at high levels of chlamydial organism load, but is not evident at lower levels of organism load [12]. This raises the question of whether other treatments are needed or if extended doses of azithromycin are needed to effectively treat rectal chlamydia infection with a high organism load [20].

Reinfection was also associated with higher organism load in the index infection. Similar results were reported by Geisler et al. [21] who reported higher inclusion-forming units in men who reported a previous chlamydia infection and suggested that the sampling time relative to the time of acquiring infection may be a potential bias as samples taken later in an infective cycle are likely to have higher organism loads compared to sampling early after infection.

In our study genovars $G, D$ and $J$ were the most prevalent in MSM and the genovar distribution was similar to the general (non-anatomically specific) distribution in Australia [22], Sweden, The Netherlands and United States [23]. The prevalence of LGV in our study was $\sim 3 \%$ which is similar to the prevalence of $2 \cdot 4 \%$ from an Australian community setting [24], but greater than that reported in Dutch STI clinics $(1 \cdot 2 \%)$ [25] and lower than that reported in UK HIV/GUM clinics (14.2\%) [26]. Our finding of seven cases of asymptomatic LGV is consistent with current evidence that symptoms can be a poor predictor of rectal LGV [27] and data from European countries reporting that a considerable proportion of cases of
LGV may be asymptomatic at diagnosis [25, 26, 28]. Therefore in the absence of genotyping at diagnosis, cases of LGV will be missed unless routine genotyping of rectal chlamydia is performed. Last, eight cases of rectal samples were found to be genovar B, the genovar normally associated with trachoma. Genovar B at the rectal site has previously been reported [29] and may occur as absolute tissue tropism by genovar does not exist.

The strengths of our study are that we believe this is the only study that has quantified the association between chlamydia organism load in MSM with repeat rectal chlamydial infections. We also used genotyping/MLST to aid in the differentiation between treatment failure and reinfection.

There are several potential limitations to our study. First, it is possible that false-positive diagnoses may have occurred in men retested within 4 weeks after treatment. However, our sensitivity analysis found that excluding those retested within 28 days had no effect on the association between repeat positivity and organism load. Second, MLST was only able to differentiate between $20 \%$ of repeat infections with the same genovar; and it is possible that men with the same MLST profile on repeat testing could have been reinfected with the same organism rather than this representing a treatment failure. Our classification of men as either treatment failure or reinfection was based on self-reported sexual risk behaviour data which may have been inaccurate or under-reported. However, our classification of treatment failure was conservative and the sexual behaviour data from our sample was comparable to that in a nationally representative sample of MSM, which found a similar proportion reporting $\geqslant 6$ partners in the last 3 months (26\% in each case) or reporting always using a condom in the last 3 months ( $29 \%$ vs. $24 \%$ ) [30]. Until a novel immunological or molecular marker of true reinfection is available, analyses similar to ours will be subject to misclassification bias. Finally, degradation of the stored frozen samples over time [31] may have effected organism load estimates as the stored rectal samples used would have been thawed and refrozen for other studies undertaken at the clinic. We also reported organism load without taking sampling variability into consideration (i.e. not per number of eukaryotic cells present). However, it has been reported that adjusting for eukaryotic cells is inappropriate because inflammatory cells produced during an acute infection are attracted to the site of infection [32]. 


\section{CONCLUSION}

We believe this is the first study to show that higher chlamydial load is associated with repeat rectal chlamydia infection and adds to the growing evidence that chlamydial load is important to treatment outcomes at other sites. With higher organism loads increasing the risk of repeat chlamydia positivity, it remains unknown if $1 \mathrm{~g}$ azithromycin remains the most effective treatment for rectal infections.

\section{ACKNOWLEDGEMENTS}

This work was supported by an Australian National Health and Medical Research Council grant (no. 568971).

\section{DECLARATION OF INTEREST}

None.

\section{REFERENCES}

1. World Health Organization. Global incidence and prevalence of selected curable sexually transmitted infections - 2008. Geneva: WHO, 2012.

2. National Notifiable Diseases Surveillance System. Number of notifications of chlamydial infections, Australia, by age group and sex, 2014 (http://www9. health.gov.au/cda/source/cda-index.cfm). Accessed February 2015.

3. Bernstein KT, et al. Rectal gonorrhea and chlamydia reinfection is associated with increased risk of HIV seroconversion. Journal of Acquired Immune Deficiency Syndrome 2010; 53: 537-543.

4. Centers for Disease Control and Prevention. Sexually transmitted diseases treatment guidelines, 2015. Morbidity and Mortality Weekly Report 2015; 64: 1138.

5. Kong FYS, et al. The efficacy of azithromycin and doxycycline for the treatment of rectal chlamydia infection: a systematic review and meta-analysis. Journal of Antimicrobial Chemotherapy 2015; 70: 1290-1297.

6. Lanjouw E, et al. European guideline for the management of Chlamydia trachomatis infections. International Journal of STD AIDS 2010; 21: 729-737.

7. Australian Sexual Health Alliance. Australian STI management guidelines for use in primary care, 2014.

8. Walker J, et al. Chlamydia trachomatis incidence and reinfection among young women-behavioural and microbiological characteristics. PLoS ONE 2012; 7: e37778.

9. West SK, et al. Infection with Chlamydia trachomatis after mass treatment of a trachoma hyperendemic community in Tanzania: a longitudinal study. Lancet 2005; 366: 1296-1300.
10. Vodstrcil LA, et al. The epidemiology of organism load in genital Chlamydia trachomatis infection - a systematic review. Journal of Infectious Diseases 2015; 211: 1628-1645.

11. Bhengraj AR, et al. Decreased susceptibility to azithromycin and doxycycline in clinical isolates of Chlamydia trachomatis obtained from recurrently infected female patients in India. Chemotherapy 2010; 56: 371-377.

12. Wang SA, et al. Evaluation of antimicrobial resistance and treatment failures for Chlamydia trachomatis: a meeting report. Journal of Infectious Diseases 2005; 191: 917-923.

13. Stevens MP, et al. Development and evaluation of an ompA quantitative real-time PCR assay for Chlamydia trachomatis serovar determination. Journal of Clinical Microbiology 2010; 48: 2060-2065.

14. Bom R, et al. Evaluation of high-resolution typing methods for Chlamydia trachomatis in samples from heterosexual couples. Journal of Clinical Microbiology 2011; 49: 2844-2853.

15. World Health Organization. Guidelines for the management of sexually transmitted infections. Geneva: WHO. 2003.

16. Harrison JD, Jones JA, Morris DL. Azithromycin levels in plasma and gastric tissue, juice and mucus. European Journal of Clinical Microbiology and Infectious Disease 1991; 10: 862-864.

17. Kirchhoff RM, et al. Determination of azithromycin in gastric biopsy samples. International Journal of Clinical Pharmacology and Therapeutics 1999; 37: 361-364.

18. Hocking JS, et al. Treatment of rectal chlamydia infection may be more complicated than we originally thought. Journal of Antimicrobial Chemotherapy 2015; 70: $961-964$.

19. van Rooijen MS, et al. Spontaneous pharyngeal Chlamydia trachomatis RNA clearance. A crosssectional study followed by a cohort study of untreated STI clinic patients in Amsterdam, The Netherlands. Sexually Transmitted Infections 2015; 91: 157-164.

20. Horner PJ. Azithromycin antimicrobial resistance and genital Chlamydia trachomatis infection: duration of therapy may be the key to improving efficacy. Sexually Transmitted Infections 2012; 88: 154-156.

21. Geisler WM, et al. Quantitative culture of Chlamydia trachomatis: relationship of inclusion-forming units produced in culture to clinical manifestations and acute inflammation in urogenital disease. Journal of Infectious Diseases 2001; 184: 1350-1354.

22. Twin J, et al. Chlamydia trachomatis genotypes among men who have sex with men in Australia. Sexually Transmitted Diseases 2011; 38: 279-285.

23. Christerson L, et al. Chlamydia trachomatis strains show specific clustering for men who have sex with men compared to heterosexual populations in Sweden, the Netherlands, and the United States. Journal of Clinical Microbiology 2012; 50: 3548-3555.

24. Templeton DJ, et al. Chlamydia trachomatis serovars in community-based HIV-positive and HIV-negative men who have sex with men in Sydney, Australia. Sexually Transmitted Infections 2011; 87: 501-502. 
25. de Vrieze NHN, et al. Anorectal and inguinal lymphogranuloma venereum among men who have sex with men in Amsterdam, the Netherlands: trends over time, symptomatology and concurrent infections. Sexually Transmitted Infections 2013; 89: 548-552.

26. Annan N, et al. Rectal chlamydia - a reservoir of undiagnosed infection in men who have sex with men. Sexually Transmitted Infections 2009; 85: 176-179.

27. Lister Nichole A, et al. Clinical significance of questionnaire-elicited or clinically reported anorectal symptoms for rectal Neisseria gonorrhoeae and Chlamydia trachomatis amongst men who have sex with men [Erratum appears in Sex Health 2008; 5: 211]. Sex Health 2008; 5: 77-82.

28. Haar K, et al. Lymphogranuloma venereum in men screened for pharyngeal and rectal infection,
Germany. Emerging Infectious Diseases 2013; 19: 488 492.

29. Geisler WM, Morrison SG, Bachmann LH. Absence of lymphogranuloma venereum strains among rectal Chlamydia trachomatis outer membrane protein A genotypes infecting women and men who have sex with men in Birmingham, Alabama. Sexually Transmitted Diseases 2008; 35: 856-858.

30. Holt M, et al. Gay Community Periodic Surveys: National Report 2010. Sydney, 2011.

31. van Dommelen $\mathbf{L}$, et al. Influence of temperature, medium, and storage duration on Chlamydia trachomatis DNA detection by PCR. Journal of Clinical Microbiology 2013; 51: 990-992.

32. Moorman DR, Sixbey JW, Wyrick PB. Interaction of Chlamydia trachomatis with human genital epithelium in culture. Microbiology 1986; 132: 1055-1067. 